\title{
Manipulation of SMARCA2 and SMARCA4 transcript levels in porcine embryos differentially alters development and expression of SMARCA1, SOX2, NANOG, and EIF1
}

\author{
Luca Magnani and Ryan A Cabot \\ Department of Animal Sciences, Purdue University, 915 West State Street, West Lafayette, Indiana 47907, USA \\ Correspondence should be addressed to R A Cabot; Email: rcabot@purdue.edu
}

\begin{abstract}
Epigenetic reprogramming plays a pivotal role during embryogenesis, including both covalent and non-covalent modifications to chromatin. In this study, we investigated the role of SNF2 chromatin remodeling ATPases (SMARCA2 (previously known as BRAHMA), SMARCA4 (previously known as BRG1), SMARCA5 (previously known as SNF2H), SMARCA1 (previously known as SNF2L), CHD3, and CHD5) during porcine preimplantation embryonic development. Transcript levels for these ATPases change dynamically throughout development. We also investigated the effect of altering transcript levels of SMARCA2 and SMARCA4 via mRNA injection.

Overexpression of SMARCA2 and SMARCA4 severely impaired embryo development. Results from these experiments show that embryos injected with SMARCA2 mRNA arrest between the four-cell and blastocyst stages. However, embryos injected with either wild-type SMARCA4 or a dominant negative variant or SMARCA4 arrest before zygotic genome activation. No differences in transcript abundance of SOX2, POU5F1, NANOG, and EIF1 (previously known as eIF1A) were detected after injection with SMARCA2 or its dominant negative variant at $48 \mathrm{~h}$ post-injection. Conversely, embryos injected with wild-type SMARCA4 and its dominant negative variant possessed altered expression of these genes. Examination of SNF2-type ATPase transcript abundance across all treatment groups revealed that only SMARCA1 was altered following injection with wild-type SMARCA2 and wild-type and dominant negative SMARCA4. We conclude that the arrest in porcine embryo development observed after injection is specific to the ATPase injected. Our data strongly support the hypothesis that SMARCA2 and SMARCA4 play different but fundamental roles controlling gene expression during early mammalian embryogenesis.

Reproduction (2009) 137 23-33
\end{abstract}

\section{Introduction}

Epigenetic reprogramming during cleavage development is a dynamic process. Soon after fertilization, several mammalian embryos (mouse, cattle, and human) undergo global DNA demethylation to erase epigenetic marks previously accumulated during germ cell development (Monk et al. 1987, Dean et al. 2001, Fulka et al. 2004). It is likely that DNA demethylation is necessary for the re-establishment of pluripotency that occurs through the reactivation of POU5F1, NANOG, and SOX2 (Reik et al. 2001, Bortvin et al. 2003, Wuensch et al. 2007). Modifications on the histone tails are also important during cleavage development. Methylation on specific arginine residues on histone H3 mark cells in the four-cell-stage mouse embryo that will form the pluripotent inner cell mass (ICM); when histone methylation was ectopically increased on these particular arginine residues, direct up-regulation of SOX2 and NANOG transcripts was observed (Torres-Padilla et al. 2007). Covalent modifications including mono-, di-, trimethylation on the lysine 9 residue of histone $\mathrm{H} 3$ are associated only with the maternal pronucleus, and are in part responsible for the epigenetic asymmetry between the two parental genomes in the murine embryos (Liu et al. 2004, van der Heijden et al. 2005). The disturbance of epigenetic reprogramming is thought to contribute to developmental abnormalities observed in embryos produced by using somatic cell nuclear transfer (Armstrong et al. 2006).

Manipulation of the chromatin architecture is referred to as chromatin remodeling. Chromatin remodeling plays a crucial role during epigenetic reprogramming. Histones $\mathrm{H} 3$ and $\mathrm{H} 4$ and variant $\mathrm{H} 3.3$ are translated from maternal mRNA and promptly replace the sperm protamines after fertilization (Wiekowski et al. 1997). Histone H3.3 is responsible for maintaining epigenetic memory in Xenopus (Ng \& Gurdon 2008), and it has been shown that this histone variant is distributed differently between the two parental genomes, with histone H3.3 incorporated preferentially in the male 
pronucleus of mouse embryos (Torres-Padilla et al. 2006). Interestingly, H3.3 needs the chromatin remodeling factor CHD1 to be incorporated in the male pronuclei in Drosophila embryos (Konev et al. 2007). Another example of the importance of chromatin remodeling comes from a study in the mouse where the deletion of the chromatin remodeling factor Smarca4 results in the repression of a large number of genes during zygotic genome activation (ZGA; Bultman et al. 2006).

CHD1 and Smarca4 are members of a large family of enzymes called the SNF2-type ATP-dependent chromatin remodeling enzymes. They generally exist in multiprotein complexes and are able to actively rearrange nucleosomes using the energy obtained from ATP hydrolysis. These chromatin remodeling complexes are built around a core ATPase that possesses a helicase domain and various accessory domains that allow them to interact with specific histone tail modifications (Sif 2004, Choudhary \& Varga-Weisz 2007, Gangaraju \& Bartholomew 2007). In mammals, these complexes are traditionally divided into three large subfamilies depending on the accessory domains found on the ATPase subunits: SWI/SNF (bromodomain), ISWI (SANT domain), and CHD (chromodomain).

The SWI/SNF subfamily of ATP-dependent chromatin remodeling complexes uses two different ATPases (SMARCA2 (previously known as BRAHMA) and SMARCA4 (previously known as BRG1)) depending on the specific complex formed (Kwon \& Wagner 2007). SWI/SNF complexes participate in a large number of cellular processes and have been shown to be necessary for embryonic development; deletion of Smarca4 or other subunits such as SNF5/ini5, BAF60, SMARCC1, and PBRM1 are all lethal at peri-implantation stages (as reviewed by de la Serna 2006). SMARCA2 and Smarca4 share $75 \%$ identity at the amino acid level but are not functionally redundant as shown by deletion of SMARCA2. Mice that lack functional SMARCA2 do not present major abnormalities aside from a mild overgrowth phenotype (Bultman et al 2000).

ATP-dependent chromatin remodeling is important for mammalian cleavage development. The objective of the present study was to investigate the pattern of expression of six other core ATPases (SMARCA4, SMARCA2, SMARCA5 (previously known as SNF2H), SMARCA1 (previously known as SNF2L), CHD3, and CHD5) belonging to the SNF2 family of chromatin remodelers in the porcine embryo, and to assess the effects of SMARCA2 and SMARCA4 misregulation on porcine embryo development. We hypothesized that altering levels of SMARCA2 and SMARCA4 will interfere with the regulation of key genes involved in ZGA and the maintenance of pluripotency and also perturb the expression of other SNF2-type chromatin remodeling ATPases.

\section{Results}

\section{SNF2-type ATPases are dynamically regulated through porcine cleavage development}

SMARCA4 and SMARCA2, the SWI/SNF ATPase subunits, are differentially expressed through cleavage development in porcine embryos (Fig. 1A and B). SMARCA4 mRNA levels are at first downregulated in MII oocytes (-16-fold, germinal vesicle $(\mathrm{GV})$ versus $\mathrm{MII}, P<0.05)$. SMARCA4 transcript levels then return to levels similar to those found in GV oocytes at the two-cell stage and do not change afterward (GV versus 2C, 4C, 8C, and blastocyst, $P>0.05$ ) in both parthenogenetic and In vitro-fertilized (IVF) embryos. Conversely, SMARCA2 transcript levels are reduced in MII oocytes $(-12$-fold, GV versus MII, $P<0.05)$ and reach the minimum during the eight-cell and blastocyst stages (18- and 16-fold, GV versus $8 \mathrm{C}$ and $B L$ respectively $P<0.05)$ in parthenogenetic embryos. In vitro-fertilized embryos possessed a significantly lower amount of SMARCA2 only at the blastocyst stage when compared with GV-stage oocytes (minus fourfold, GV versus blastocyst, $P<0.05$ ).

Transcripts for the ISWI ATPases, SMARCA5 and SMARCA1, were also examined in cleavage-stage embryos (Fig. 1C and D). In embryos obtained by IVF, transcripts for SMARCA5 were increased at the two- and four-cell stages when compared with MII oocytes (3- and 4 -fold, MII versus $2 \mathrm{C}$ and $4 \mathrm{C}$ respectively, $P<0.05)$ and then reduced at the eight-cell and blastocyst stages (-1.9- and -3.8 -fold, GV versus $8 \mathrm{C}$ and blastocyst

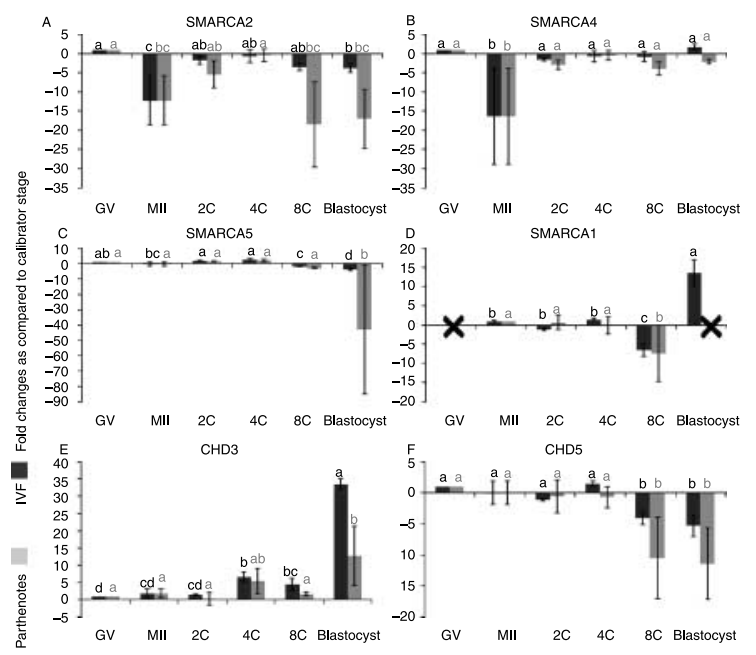

Figure 1 Relative abundance of SNF2-type ATPase in porcine oocytes and cleavage embryos. The pattern of expression of SNF2-type ATPases changes throughout cleavage development. Different subscripts represent significant differences across development stages within each treatment after Tukey's multiple comparison post-test $(P<0.05)$. Fold differences were calculated with respect to the GV (or MII for SMARCA1)-stage oocyte. The results shown here are the average of three independent experimental replicates. Bars represent S.D. Treatments are labeled as follows: in vitro-fertilized embryos (IVF) and parthenogenetic embryos (parthenotes). 
respectively, $P<0.05)$. In parthenogenetic embryos, SMARCA5 transcripts were dramatically decreased at the blastocyst stage $(-42$-fold, GV versus $\mathrm{BL}, P<0.05)$. Interestingly, SMARCA1 transcripts were not detectable in either GV-stage oocytes or parthenogenetic blastocyst-stage embryos. SMARCA1 transcripts were present in low abundance in mature oocytes and further downregulated at the eight-cell stage (minus sevenfold, MII versus $8 C, P<0.05)$. SMARCA1 was downregulated also at the eight-cell stage in IVF embryos (minus sixfold, MII versus $8 \mathrm{C}, P<0.05)$. Surprisingly, we found SMARCA1 transcripts to be present in higher abundance in IVF-derived blastocyst-stage embryos (13-fold, MII versus blastocyst, $P<0.05$ ).

Finally, we examined the expression patterns of $\mathrm{CHD} 3$ and CHD5, two ATPases of the CHD family (Fig. 1E and F). Embryos produced by IVF showed increased $\mathrm{CHD} 3$ transcripts at the four-cell stage (6 fold, GV versus 4C, $P<0.05)$ and then at the blastocyst stage (33-fold, GV versus $\mathrm{BL}, P<0.05)$. Parthenogenetic embryos had a fivefold increase in $\mathrm{CHD} 3$ transcript levels at the fourcell stage, although not statistically significant, and 13fold increase at the blastocyst stage (13-fold, GV versus blastocyst, $P<0.05)$. CHD5 transcripts were in lower abundance in the eight-cell- and blastocyst-stage embryos obtained by IVF $(-4$ and -5 fold, GV versus $8 \mathrm{C}$ and blastocyst respectively, $P<0.05)$. In parthenogenetic embryos, CHD5 transcripts were found in lower abundance in the eight-cell and blastocyst-stage embryos ( -10 - and -12 -fold, GV versus $8 \mathrm{C}$ and blastocyst respectively, $P<0.05)$.

\section{Relative transcript abundance of SNF2-type ATPases at different time points in porcine cleavage development}

The amount of SNF2 ATPases transcripts is relative to the specific developmental stage (Fig. 2). In the GV-stage oocytes, SMARCA5 ATPase transcripts were present in the highest abundance (SMARCA5 versus all others, $P<0.05$ ). SMARCA2 and SMARCA4 transcripts were present at similar levels, while CHD3 was in extremely low abundance; SMARCA1 transcripts were not detectable. This profile is similar to that found in MII oocytes, where SMARCA5 and CHD5 were again the most abundant ATPases $(P<0.05)$. SMARCA2 and SMARCA4 were present at similar levels; CHD3 and SMARCA1 were the least abundant transcripts at this stage.

In IVF-derived embryos at the two-cell stage, we found SMARCA5 to be in the highest abundance; CHD5 transcripts were also highly represented while SMARCA2 and SMARCA4 transcripts were expressed at similar levels. Also at this stage, SMARCA1 and CHD3 transcripts continued to be present at low levels. During the fourcell stage, SMARCA5 remained present in the highest abundance, but SMARCA2 and SMARCA4 transcripts were expressed at levels similar to CHD5 (SMARCA2 and SMARCA4 versus CHD5, P>0.05); SMARCA1 and

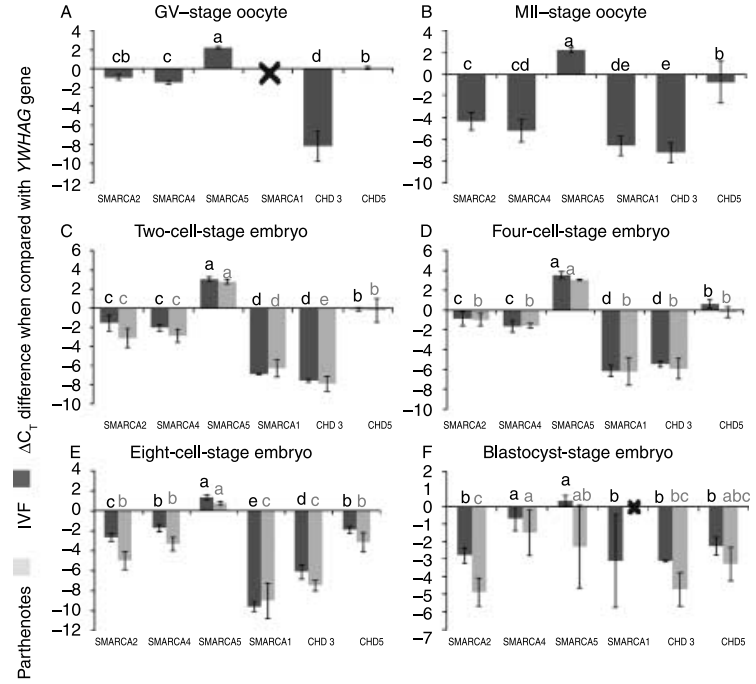

Figure 2 Relative abundance of SNF2-type ATPase at a defined stage of porcine development. The pattern of expression of SNF2-type ATPases is defined at each stage of cleavage development. Different subscripts represent significant differences across SNF2-type ATPase transcript levels within each treatment after Tukey's multiple comparison post-test $(P<0.05) . \Delta C_{\mathrm{T}}$ values were calculated with respect to the $Y W H A G$ transcript levels. The results shown here are the average of three independent experimental replicates. Bars represent S.D. Treatments are labeled as follows: in vitro-fertilized embryos (IVF) and parthenogenetic embryos (parthenotes).

CHD3 were again in the lowest abundance. At the eightcell stage, SMARCA5 was in the highest abundance, followed by SMARCA4 and CHD5. Also at the eight-cell stage, SMARCA2 transcripts were detected at an intermediate level and SMARCA1 and CHD3 transcripts were present at low levels. In the blastocyst-stage embryos, we found SMARCA4 and SMARCA5 transcripts to be present in the highest abundance. All the other ATPases were found to be present at similar levels.

We found that embryos obtained by parthenogenesis possessed a pattern of expression which closely resembled that observed in IVF embryos. Exceptions to this were found at the blastocyst stage. Parthenogenetic embryos had a lower amount of SMARCA5 and did not possess transcripts for SMARCA1.

\section{Ectopic expression of SMARCA2 and SMARCA4 lead to arrested development in porcine parthenogenetic embryos}

No significant differences were detected in the proportion of embryos that progressed to the four-cell stage across treatment groups (injected with SMARCA2-WT, SMARCA2-DN, GFP, and non-injected; data not shown). When we ectopically expressed wild-type SMARCA4 or dominant negative SMARCA4 in parthenogenetic embryos, we observed a more dramatic phenotype (Table 1). The dominant negative mutations consist of a single amino acid mutation that inactivates the ATPase 
Table 1 In vitro developmental potential of porcine parthenogenetic embryos injected with in vitro-synthesized SMARCA4 mRNA.

\begin{tabular}{lccrr}
\hline Treatment & \% Blastocyst & \% Cleaved & \% Four-cell stage & Average nuclei number \\
\hline SMARCA4-WT & $1.8^{\mathrm{a}}$ & $10.6^{\mathrm{a}}$ & $6.9^{\mathrm{a}}$ & $1.4 \pm 4.9^{\mathrm{a}}$ \\
SMARCA4-DN & $4.1^{\mathrm{a}}$ & $28.6^{\mathrm{b}}$ & $18.3^{\mathrm{b}}$ & 234 \\
GFP & $28.6^{\mathrm{b}}$ & $53.3^{\mathrm{c}}$ & $67.2^{\mathrm{c}}$ & $2.9 \pm 6.5^{\mathrm{b}}$ \\
Non-injected & $32.8^{\mathrm{b}}$ & $52.9^{\mathrm{c}}$ & $67.7^{\mathrm{c}}$ & $10.9 \pm 13.3^{\mathrm{c}}$ \\
\hline
\end{tabular}

All embryos were included in the analysis presented here; degenerated embryos were considered as embryos with zero cells for the purposes of determining cell number. Different superscripts indicate differences at $P<0.001$. Treatments are labeled as follows: wild-type SMARCA4 (SMARCA4-WT), dominant negative SMARCA4 (SMARCA4-DN), GFP (GFP), and non-injected control (non-injected).

activity without altering complex formation (Muchardt \& Yaniv 1993, Dunaief et al. 1994). Embryos injected with wild-type SMARCA4 tended to cleave at a lower rate and reach the four-cell stage with a significantly lower frequency when compared with embryos injected with dominant negative SMARCA4 $(10.6 \%$ and $6.9 \%$ vs $28.6 \%$ and $18.3 \%$, SMARCA4-WT and SMARCA4-DN versus GFP and non-injected respectively, $P<0.05$ ). Embryos injected with SMARCA4-WT and SMARCA4$\mathrm{DN}$ had a significantly lower developmental potential as shown by blastocyst formation and nuclei number $(1.8 \%$ and $1.4 ; 4.1 \%$ and 2.9 vs $28.6 \%$ and $10.9 ; 32.8 \%$ and $10.5 \%$; SMARCA4-WT and SMARCA4-DN versus GFP and non-injected respectively, $P<0.05)$.

\section{Ectopic expression of SMARCA2 and SMARCA4 specifically alters gene expression}

While ectopic overexpression of SMARCA2 did not have any significant effect $48 \mathrm{~h}$ post-injection on the expression of POU5F1, NANOG, and eukaryotic elongation initiation factor 1 (EIF1; previously known as elF1A), the dominant negative form of SMARCA2 led to an increased amount of SOX2 transcripts (4-fold, DN versus $\mathrm{WT}, P<0.05$; Figs $3 \mathrm{~A}$ and $4 \mathrm{~A}$ ). Furthermore, embryos injected with the wild-type form of SMARCA2 showed an increased amount of SMARCA1 (10-fold, WT versus non-injected control, $P<0.05)$.

Embryos injected with SMARCA4 or its dominant negative variant had perturbed gene expression $48 \mathrm{~h}$ post-injection. Transcriptional levels of SOX2 were statistically lower in abundance in embryos injected with wild-type SMARCA4 when compared with the other treatment groups $(-10-$ fold, WT versus all other treatments, $P<0.05$ ). While POU5F1 expression was not perturbed, we could not detect NANOG transcripts in embryos injected with wild-type SMARCA4. Injection with dominant negative SMARCA4 resulted in a statistically lower amount of NANOG ( -5 -fold, DN versus GFP and non-injected control, $P<0.05)$. EIF1, a marker gene for ZGA, was also misregulated: injection of either wild-type or dominant negative SMARCA4 resulted in lower transcript levels for EIF1 (-7- and -11-fold, SMARCA4WT and SMARCA4-DN versus GFP and non-injected control respectively, $P<0.05)$. We were able to detect an increased amount in SMARCA4 transcript both in the wild-type and in the dominant negative treatments (13- and 11-fold, SMARCA4-WT and SMARCA4-DN versus GFP and control respectively, $P<0.05)$. We also found a 5 -fold increase in SMARCA1 transcript in both SMARCA4-WT and SMARCA4-DN treatments (5-fold, SMARCA4-WT and SMARCA4-DN versus GFP and noninjected control, $P<0.05)$.

\section{Discussion}

Non-covalent modifications of chromatin may have a significant impact on the embryonic transcription profile and influence embryo development. Alterations of the chromatin structure often accompany transcriptional regulation and require the activity of complexes able to alter the nucleosome array. Few studies have addressed the dynamics of chromatin remodeling during

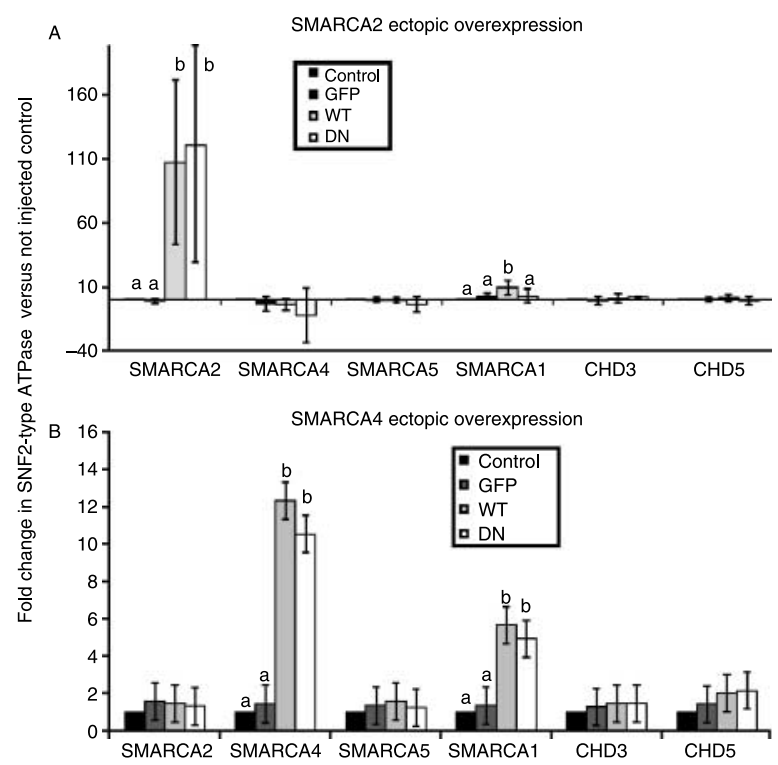

Figure 3 Embryos injected with wild-type or dominant negative SMARCA2 or SMARCA4 have altered expression of SNF2-type ATPases. Embryos injected with the SWI/SNF ATPases SMARCA2 or SMARCA4 possess altered levels of other SNF2-type ATPases $48 \mathrm{~h}$ post-injection. Different subscripts represent significant differences in transcript levels across treatments within each treatment after the LSD multiple comparison post-test $(P<0.05)$. Fold differences were calculated using non-injected control as the calibrator. The results shown here are the average of three independent experimental replicates. Bars represent s.D. Treatments are labeled as follows: wild-type ATPase (WT), dominant negative ATPase (DN), GFP (GFP), and non-injected control (control). 


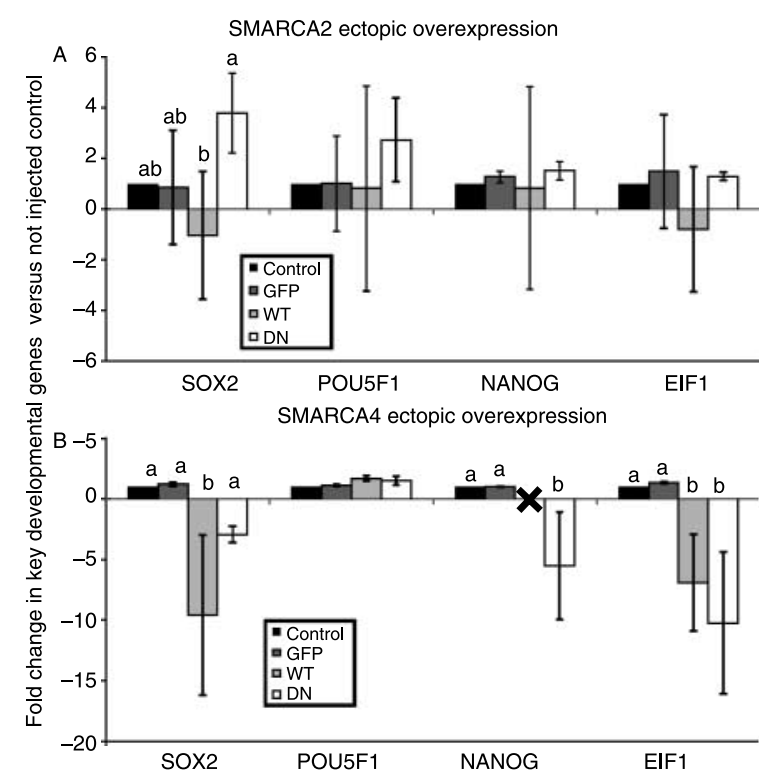

Figure 4 Embryos injected with wild-type or dominant negative SMARCA2 or SMARCA4 have altered gene expression. Embryos injected with the SWI/SNF ATPases SMARCA2 or SMARCA4 possess altered levels of key developmental genes $48 \mathrm{~h}$ post-injection. Different subscripts represent significant differences in transcript levels across treatments within each treatment after the LSD multiple comparison post-test $(P<0.05)$. Fold differences were calculated using a noninjected control as the calibrator. The results shown here are the average of three independent experimental replicates. Bars represent S.D. Treatments are labeled as follows: wild-type ATPase (WT), dominant negative ATPase (DN), GFP (GFP), and non-injected control (control).

mammalian embryogenesis. Here, we show that misregulation of the catalytic ATPases of the SWI/SNF family results in perturbed embryonic development possibly via misregulation of other key developmental genes.

In regard to the relative abundance of SNF2 ATPase transcripts in mammalian embryos, our data demonstrate that a species-specific difference exists between the murine model and other mammals. In mouse embryos, Smarca2 transcripts are downregulated after the two-cell stage and become almost undetectable from the four-cell stage until the blastocyst stage, whereas Smarca2 transcripts become more abundant (LeGouy et al. 1998, Bultman et al. 2000). LeGouy noted that SMARCA2 protein becomes restricted to the ICM at the blastocyst stage. Interestingly, they also found that ES cells (which are derived from the ICM) did not possess SMARCA2, but they express Smarca2 upon differentiation (LeGouy et al. 1998). On the other hand, Smarca4 transcripts are in relatively high abundance during mouse cleavage development (LeGouy et al. 1998). We showed that in parthenogenetic porcine embryos and those produced by IVF, SMARCA2 is expressed at a comparable level with SMARCA4 during most of cleavage development. SMARCA2 is then downregulated at the blastocyst stage (Figs 1 and 2). This profile is similar to the one observed in primate embryos
(Zheng et al. 2004), suggesting that the pig embryo may represent a better model for human cleavage-stage embryo development in studies involving chromatin remodeling when compared with the mouse embryo.

Similarly to the SWI/SNF ATPases (SMARCA4 and SMARCA2), the ISWI ATPases (SMARCA5 and SMARCA1) are $75 \%$ identical at the nucleotide level and share $81 \%$ of their amino acid sequence. Studies in the mouse have shown that SMARCA5 deletion leads to post-implantation lethality (Stopka \& Skoultchi 2003). The expression pattern of SMARCA5 has not been extensively investigated in cleavage-stage embryos. SMARCA5 was present in high abundance as maternal mRNA and then degraded at the eight-cell stage in rhesus monkey embryos (Stopka \& Skoultchi 2003). Interestingly, SMARCA1 mRNA was barely detectable throughout cleavage development in the rhesus monkey (Zheng et al. 2004), similar to our findings that SMARCA1 transcripts are not detectable in the GV-stage porcine oocytes and parthenogenetic blastocyst-stage porcine embryos and are in extremely low abundance at all other stages of development examined (Figs 1 and 2).

The third family of SNF2-type chromatin remodeling complexes is represented by the CHD complexes (also known as Nurd or Mi-2 in human; Bowen et al. 2004, Hall \& Georgel 2007). Relatively less is known about the function of the CHD complexes and no studies have been conducted to investigate their expression pattern in mammalian embryos, although it has been shown that CHD2 is essential for embryo development (Marfella et al. 2006). We found that CHD3 and CHD5 were expressed at differing levels until the blastocyst stage, where they are present in similar abundance. Our data, taken collectively, show that chromatin remodeling may be regarded as a fine-tuned process in cleaving embryos, with many ATPases that can form several alternative complexes in order to interact with specific loci at specific times of development. More experiments are needed to address the protein localization of such ATPases during embryo development.

We expected to observe a detrimental effect following SMARCA4 dominant negative injection, since it may outcompete the endogenous maternal SMARCA4, mimicking a knock-out phenotype. Functional maternal SMARCA4 is necessary for ZGA in mouse embryos; ablation of SMARCA4 resulted in the downregulation of $\sim 30 \%$ of $\alpha$-amanitin-sensitive genes (Bultman et al. 2006). This explains why embryos injected with dominant negative SMARCA4 tend to arrest at the fourcell stage (Table 1), at the same stage when ZGA occurs in the pig (Jarrell et al. 1991). This is also consistent with the downregulation of EIF1 observed following the injection of dominant negative SMARCA4. Transcripts for EIF1 are a good marker for ZGA in many species (Latham et al. 1992, De Sousa et al. 1998). Our results support the hypothesis that SMARCA4 is important for the onset of ZGA in pig embryos. 
Conversely, we did not expect the dramatic developmental arrest observed in embryos injected with the wild-type SMARCA4. The dynamics of the arrest, with the majority of embryos blocking before the two-cell stage, led us to hypothesize that excess SMARCA4 may have interfered with the first cleavage division. SMARCA4 has been linked extensively to the control of the cell cycle (as reviewed in Muchardt \& Yaniv 2001). It is possible that the arrest comes from the antiproliferative properties of SMARCA4 in association with the tumor suppressor protein retinoblastoma ( $\mathrm{pRb}$; Dunaief et al. 1994). The $\mathrm{pRb}$ protein is one of the major cell cycle regulators that control the G1/S transition as well as progression through $\mathrm{S}$ phase. $\mathrm{pRb}$ and SMARCA4 form a repressor complex (Dunaief et al. 1994). Co-expression of SMARCA4 and pRb in human C33a cells (a cell line that does not possess SMARCA4) led to cell cycle arrest (Zhang et al. 2000). SMARCA4 overexpression led to pRb-mediated growth arrest also in SMARCA4/ SMARCA2 lacking human SW13 cells (Dunaief et al. 1994, Shanahan et al. 1999, Strobeck et al. 2000). Interestingly, forced ectopic expression of SMARCA4 in U2OS cells (cells that do possess SMARCA4) led to growth arrest as well (Zhang et al. 2000). pRb protein is present at the two-cell stage during zygotic gene activation in the mouse (Xie et al. 2005). The SMARCA4-pRb block is mediated by cyclin A and $\mathrm{E}$ degradation. It will be interesting to analyze the expression pattern of these two cyclin molecules in embryos injected with wild-type and dominant negative variants of SMARCA4. The point mutation in the dominant negative variant of SMARCA4 is outside the loop involved in pRb-SMARCA4 contact, so we assume that dominant negative SMARCA4 can still form the SWI/SNF complex. Our data nonetheless suggest that functional SMARCA4 is required to arrest the cell cycle, since embryos injected with dominant negative SMARCA4 have a significantly different phenotype (Table 1).

It is important to note that the data shown in Table 1 include all injected embryos that did not lyse immediately after microinjection. Degenerated and fragmented embryos scored for cell number at $144 \mathrm{~h}$ post-activation, the time when in vitro-produced porcine embryos will have reached the blastocyst stage of development, were considered as embryos with zero cells. By scoring embryo development in this manner, we include all embryos in our analysis, but greatly increase the range of values for the average cell number in each treatment group. It is important to note that the group injected with wild-type SMARCA4 had a significantly lower average number of nuclei per embryo even when compared with the group injected with dominant negative SMARCA4. This was largely due to arrest of embryos injected with SMARCA4 at an early stage of cleavage, thereby lowering the number of embryos that progressed through development.
Retinoblastoma protein $\mathrm{pRb}$ is able to form a complex with SMARCA2 as well. Interestingly, pRb, SMARCA2, and HDAC1 are responsible for the formation of the repressor protein HP1 $\beta$ heterochromatin foci in aging human melanocyte cells (Bandyopadhyay et al. 2007). SMARCA2, like SMARCA4, is able to arrest growth in SMARCA2- and SMARCA4-deficient cells in a pRb-dependent fashion (Strober et al. 1996, Trouche et al. 1997, Reisman et al. 2002). Curiously, we did not observe the same dramatic arrest of embryo development as for SMARCA4 overexpression. This suggests that SMARCA2 and SMARCA4 have distinct roles in cell cycle control during early embryogenesis. SMARCA2 may be less important during the first cleavage divisions, as demonstrated by mouse knock-out models (Bultman et al. 2000). Given that at $48 \mathrm{~h}$ post-injection, we were able to detect a 10 -fold increase in SMARCA2 mRNAs, but only a tenfold increase in SMARCA4 transcripts, lends support to this hypothesis. These two genes may be differentially translated, since similar concentrations of each mRNA were injected in all treatments groups. Thus, mammalian embryos may rely more heavily on SMARCA4 than SMARCA2 during cleavage development.

Ectopic SMARCA4 may also be integrated in other complexes with repression functions. SMARCA4 has been physically associated with six different complexes, including the mSin3A/HDAC co-repressor complex (Sif et al. 2001, Kuzmichev et al. 2002, Pal et al. 2003) and the $\mathrm{N}$-CoR complex (Underhill et al. 2000). Both these complexes possess histone deacetylases (HDAC1 and HDCA2 in mSin3A, HDAC3 in nCoR). Histone deacetylation has been linked extensively with repressed chromatin (reviewed in Hildmann et al. 2007). Histone deacetylase transcripts (HDCA2) have been detected in pig embryos as early as the four-cell stage (earlier stages were not assessed; Kumar et al. 2007). Bovine and mouse embryos possess all three HDACs as maternal transcripts in the GV- and metaphase II-stage oocytes and two-cell-stage embryos (McGraw et al. 2003, Inoue et al. 2006). Transcripts for the three deacetylases were also detected in rhesus monkey embryos, with HDAC2 being predominant. The bromodomain in SMARCA4 can interact with acetylated lysine residues on histone proteins (reviewed in Mujtaba et al. 2007). It is possible that exogenous SMARCA4 targets mSin3A/HDAC and nCoR complexes onto specific targets and repress transcription in an ATPase-dependent way.

The transcriptional repression of NANOG after SMARCA4 injection further suggests that histone deacetylation may be involved. After retinoic acid induced differentiation of mouse embryonic stem (ES) cells, NANOG expression is shut down by $\mathrm{p} 53$ through mSin3A deacetylase activity (Lin et al. 2005). Moreover, Hattori et al. (2007) showed that NANOG is repressed in trophectoderm (TE) cells and expressed in ES cells. Histones $\mathrm{H} 3$ and $\mathrm{H} 4$ were hypoacetylated in the NANOG promoter region in TE cells, correlating with its 
repressive state, while the same region in ES cells contained hyperacetylated histones. This suggests a mechanism by which wild-type SMARCA4 represses NANOG expression at $48 \mathrm{~h}$ post-injection. NANOG is typically activated during this period in porcine embryos (Magnani \& Cabot 2008). SMARCA4 may recruit the $\mathrm{mSin} 3 \mathrm{~A} / \mathrm{HDAC}$ complex onto the NANOG promoter and actively repress its transcription.

SOX2 transcripts were also repressed, although at a lower extent, in embryos injected with wild-type SMARCA4. There is evidence that histone acetylation on the SOX2 promoter is responsible for its inducible activation or silencing (Ruau et al. 2008, Sikorska et al. 2008). Moreover, the injection of a dominant negative variant of SMARCA4 or a SMARCA4 morpholino in Xenopus embryos led to increased cell proliferation and expansion of SOX2-positive cells (Seo et al. 2005), suggesting that SMARCA4 is required for SOX2 control and cell proliferation.

Ectopic expression of SMARCA2 in porcine embryos leads to a reduction in blastocyst formation; however, these embryos developed to the four-cell stage equally to the non-injected control (data not shown). Interestingly, at $48 \mathrm{~h}$ post-injection, we were able to detect a significant increase in SOX2 expression in the group injected with the dominant negative variant of SMARCA2. This indicates that functional SMARCA2 may be necessary to control SOX2 expression. However, this seems to be in contrast with the observation that functional SMARCA2 is required to activate SOX2 in rat neural cells (Kondo \& Raff 2004). It is also possible that SOX2 transcription is differently regulated in the cleavage embryo when compared with neural cells.

Injection of wild-type SMARCA2 resulted in a tenfold increase in SMARCA1. SMARCA1 transcripts are present at extremely low concentrations during development, and further decrease around the eight-cell stage. Interestingly, parthenogenetic porcine embryos do not possess detectable SMARCA1 transcripts at the blastocyst stage. SMARCA1 knock-out mice have not been reported. The increase in SMARCA1 may be detrimental at this stage of development. It would be interesting to monitor the amount of SMARCA1 at later stages following the injection of wild-type SMARCA2, as well as to directly overexpress SMARCA1. It is interesting to note that SMARCA1 is associated with differentiation in multiple cell lines (LeGouy et al. 1998, Lazzaro \& Picketts 2001, Lazzaro et al. 2006, Itoh et al. 2008). Thus, it is intriguing to speculate that a connection exists between SWI/SNF and ISWI ATPases may then exist in relation to the differentiation process (SMARCA4/ SMARCA5 to SMARCA2/ SMARCA1). SMARCA4 and SMARCA5 appear to be present at the same actively transcribed foci in early mouse embryos at the time of ZGA (Torres-Padilla \& Zernicka-Goetz 2006).

Human SMARCA2 and SMARCA4 have been shown to be involved in splicing processes. Interestingly, SMARCA2, but not SMARCA4 has been shown to be necessary for splicing of SWI/SNF target genes such as CD44 and e-cadherin in an ATPase-independent fashion (Batsché et al. 2006). SMARCA4 and SMARCA2 were shown to be directly linked to the methylation status at the same loci, suggesting that SWI/SNF complexes may exert activation/ repression functions in one of several ways (Banine et al. 2005). SMARCA2, and possibly SMARCA4, also controls splicing variants for the telomerase reverse transcriptase gene via interaction with p54 (Ito et al. 2008). At this point, we cannot exclude that the phenotype observed in our experiments is a direct effect of mis-splicing events of essential transcripts.

The results from our studies presented here were obtained using porcine embryos derived from parthenogenetic activation and IVF. Porcine embryos produced in vitro are known to possess reduced developmental competence, when compared with embryos produced in vivo. Although polyspermy is one major challenge in producing porcine embryo by using IVF, the protocols used by our laboratory have been reported to lead to term development (Abeydeera \& Day 1997, Abeydeera et al. 1998). In addition, although parthenogenetic embryos cannot develop to term, they represent a robust model for molecular events occurring during preimplantation development. Our group has recently shown that the regulation of fundamental genes such as POU5F1, NANOG, and SOX2 is closely recapitulated in embryos produced by parthenogenetic activation or IVF when compared with in vivo-derived embryos (Magnani \& Cabot 2008).

Our data taken collectively show that chromatin remodeling may be regarded as a fine-tuned process in the cleavage-stage mammalian embryos, with many ATPases that can form several alternative complexes in order to interact with specific loci at specific times of development. Our data show that overexpression of SMARCA2 and SMARCA4, the two highly similar ATPases of the SWI/SNF complex, results in different phenotypes suggesting different roles for the two proteins during porcine cleavage development. We demonstrated that alterations in the expression of SMARCA2 and SMARCA4 perturb developmentally important genes and result in the disruption of embryo development. In conclusion, our data reinforce the idea that chromatin remodeling has a central role during embryonic development via regulation of gene expression.

\section{Materials and Methods}

\section{Oocyte collection}

All chemicals were obtained from Sigma Chemical Company, unless stated otherwise. Porcine (Sus scrofa) ovaries from prepubertal gilts were collected at a local slaughterhouse and transported to the laboratory in an insulated container at $37^{\circ} \mathrm{C}$. Antral follicles between 3 and $6 \mathrm{~mm}$ in diameter were aspirated manually with a disposable $10 \mathrm{cc}$ syringe and an 18 gauge 
needle. Follicular fluid was pooled and allowed to settle by gravity. Cumulus-oocyte complexes (COCs) were resuspended in HEPES-buffered medium containing $0.01 \%$ polyvinyl alcohol (PVA; Abeydeera et al. 1998). Under a dissecting microscope, COCs with multiple layers of intact cumulus cells were selected for the experiments. For GV-stage oocytes used in PCR studies, COCs were vortexed in $0.1 \%$ hyaluronidase in HEPES-buffered medium for $6 \mathrm{~min}$ to remove the cumulus cells.

\section{In vitro maturation}

50-75 COCs were cultured in $500 \mu \mathrm{l}$ tissue culture medium 199 (TCM-199; Gibco BRL) containing 0.14\% PVA, $10 \mathrm{ng} / \mathrm{ml}$ epidermal growth factor, $0.57 \mathrm{mM}$ cysteine, $0.5 \mathrm{IU} / \mathrm{ml}$ porcine $\mathrm{FSH}$, and $0.5 \mathrm{IU} / \mathrm{ml}$ ovine $\mathrm{LH}$. COCs were matured for $42-44 \mathrm{~h}$ at $39{ }^{\circ} \mathrm{C}, 5 \% \mathrm{CO}_{2}$ in air, and $100 \%$ humidity (Abeydeera et al. 1998). Matured COCs were then vortexed in $0.1 \%$ hyaluronidase in HEPES-buffered medium containing $0.01 \%$ PVA for $4 \mathrm{~min}$ to remove the cumulus cells.

\section{Parthenogenetic activation}

Mature oocytes were artificially activated as described previously (Magnani \& Cabot 2007), by placing mature oocytes in activation medium $(300 \mathrm{mM}$ mannitol, $0.1 \mathrm{mM} \mathrm{CaCl}$, $0.1 \mathrm{mM} \mathrm{MgSO}_{4}, 0.5 \mathrm{mM}$ HEPES, $0.01 \%$ BSA) between two platinum electrodes in an Electrocell chamber and given two DC pulses of $1.2 \mathrm{kV} / \mathrm{cm}$ for $30 \mu \mathrm{s}$ provided by a BTX ElectroCell Manipulator 200 (BTX, San Diego, CA, USA).

\section{IVF}

Mature denuded oocytes were placed in a modified Tris-buffered medium (mTBM) and fertilized according to an established protocol (Abeydeera \& Day 1997), using fresh extended boar semen. Briefly, boar semen was extended in Modena Boar Semen Extender (Swine Genetics International, Cambridge, IA, USA) and kept at $17.5^{\circ} \mathrm{C}$ for up to 3 days. Before fertilization, $1 \mathrm{ml}$ extended semen was mixed with $10 \mathrm{ml}$ Dulbecco's phosphate-buffered saline (DPBS) and centrifuged at $1000 \mathrm{~g}, 25^{\circ} \mathrm{C}$, for $4 \mathrm{~min}$. Sperm were washed thrice with DPBS. The final concentration of sperm used in the fertilization experiments was $5 \times 105$ cells/ml. Groups of 30-35 oocytes were placed in $100 \mu \mathrm{lmTBM}$; gametes were co-incubated for $5 \mathrm{~h}$ at $39{ }^{\circ} \mathrm{C}$ and $5 \% \mathrm{CO}_{2}$.

\section{Embryo culture and collection}

Porcine zygote medium 3 (PZM3) containing $3 \mathrm{mg} / \mathrm{ml}$ fatty acid-free BSA was used to culture all embryos (Yoshioka et al. 2002). The embryos were maintained at $39^{\circ} \mathrm{C}, 5 \% \mathrm{CO}_{2}$, and $100 \%$ humidity until the appropriate cell stage. IVF embryos were collected as follows: two-cell ( $24 \mathrm{~h}$ post-IVF), four-cell (48 h post-IVF), eight-cell (72 h post-IVF), and blastocyst stages (day 6 post-IVF). Parthenogenetic embryos (PA) were collected as follows: two-cell ( $24 \mathrm{~h}$ post-activation), four-cell ( $48 \mathrm{~h}$ postactivation), eight-cell (72 $\mathrm{h}$ post-activation), and blastocyst stages (day 6 post-activation). Only embryos with the correct morphology at the above-indicated time points were used.

\section{Microinjection}

The sequence of human SMARCA2, SMARCA4, and their dominant negative variants were a generous gift from $\mathrm{Dr}$ Anthony Imbalzano (University of Massachusetts Medical School, Worcester, MA, USA; de La Serna et al. 2000). Messenger RNA was prepared as described previously (Magnani \& Cabot 2007). In vitro-produced mRNA was run on a denaturing agarose gel to verify size and integrity. Prior to microinjection, activated oocytes were divided randomly into four groups and placed in HEPES-buffered medium supplemented with $3 \mathrm{mg} / \mathrm{ml}$ BSA. For SMARCA2 studies, the four groups were as follows: non-injected controls, GFP mRNA injected, wild-type SMARCA2 mRNA and GFP mRNA injected (SMARCA2-WT), and dominant negative SMARCA2 mRNA and GFP mRNA injected (SMARCA2-DN). For SMARCA4 studies, the four groups were as follows: non-injected controls, GFP mRNA injected, wild-type SMARCA4 mRNA and GFP mRNA injected (SMARCA4-WT), and dominant negative SMARCA4 mRNA and GFP mRNA injected (SMARCA4-DN). Messenger RNA concentrations were $\mu \mathrm{g} / \mu \mathrm{l}$; equal amount of ATPase RNAs and GFP were mixed before injection. GFP mRNA co-injected to verify the integrity of injected mRNA translation of GFP demonstrated the quality of the mRNA sample injected. In vitro-synthesized mRNA was loaded into a sterile injection capillary attached to an Eppendorf Femtojet microinjector (Westbury, NY, USA); $\sim 8 \mathrm{pl}$ of each respective mRNA was injected into the activated oocytes. Observance of movement of the cytoplasm was used as a reference for successful injection. Embryos that lysed immediately following microinjection were discarded and not included in the analysis. After injection, the embryos were transferred into PZM3 (Yoshioka et al. 2002) embryo culture medium and cultured for 6 days (to evaluate embryo development) or cultured for $48 \mathrm{~h}$ for mRNA isolation. On day 6 , the embryos were stained with Hoechst 33342 and nuclei were counted under an epifluorescent microscope.

\section{Hoechst staining and nuclei count}

Blastocysts were collected at $144 \mathrm{~h}$ post-activation and after injection and placed in HEPES-buffered medium containing $2 \mu \mathrm{g} / \mathrm{ml}$ Hoechst 33342. Embryos were stained for $15 \mathrm{~min}$ and mounted on microscope slides. Nuclei were visualized on an epifluorescent microscope. To obtain a developmental score, the nuclei were counted (degenerate embryos counted as 0 nuclei) and averages were obtained for each treatment.

\section{Primers for real-time PCR}

Primers were designed on porcine sequences highly similar to human transcripts. Sequences were submitted to GenBank with the following accession numbers: SMARCA4 (EU780789), SMARCA2 (EU780788), SMARCA5 (EU780790), SMARCA1 (EU780791), CHD3 (EU780792), and CHD5 (EU780793). Primers for YWHAG, POU5F1, NANOG, SOX2, and all the SNF2-type ATPases were described previously (Magnani \& Cabot 2008, Magnani et al. 2008); EIF1 primers were as follows: forward, ggtgttcaaagaagatgggcaaagag and reverse, 
tttccctctgatgtgacataacttc. Transcript levels of the gene YWHAG were used to normalize transcript abundance in our assay (Magnani \& Cabot 2007).

\section{Isolation of mRNA and RT}

For the expression pattern of SNF2-type ATPases, polyadenylated mRNA was extracted from pools of 50-100 GV and MII oocytes using Dynabeads reagent (Invitrogen). Polyadenylated mRNA extracted from pools of oocytes collected on a single day was used as experimental replicate. Polyadenylated mRNA was extracted from pools of 50-100 embryos at the following stages: two-cell, four-cell, eight-cell, and blastocyst. Each mRNA extraction was replicated at least three times for each experiment. Oocytes and embryos were washed three times with PVA-PBS (nuclease free), then lysed in $100 \mu$ l lysis buffer (Invitrogen), and stored at $-80{ }^{\circ} \mathrm{C}$ until mRNA isolation. Reverse transcription was performed using the iScript kit, according to the manufacturer's instructions (Bio-Rad). For each experimental replicate, cDNA from one pool of oocytes or embryos was divided to amplify all genes during the same PCR run. For microinjected embryos, polyadenylated mRNA was extracted from 15 to 50 intact embryos $48 \mathrm{~h}$ postactivation. Each mRNA extraction was replicated at least three times for each experiment. For each experimental replicate, cDNA from one pool of oocytes or embryos was divided to amplify the six SNF2-ATPases or SOX2, POU5F1, NANOG and EIF1 genes.

\section{Quantitative real-time PCR}

A PCR Master Mix was prepared for each gene as follows: $12.5 \mu \mathrm{l}$ of $2 \times$ SYBR Green Master Mix (Bio-Rad), $5 \mu \mathrm{l}$ of $1 \mu \mathrm{M}$ forward primer, $5 \mu \mathrm{l}$ of $1 \mu \mathrm{M}$ reverse primer, and $2.5 \mu \mathrm{l}$ of cDNA. Reactions were carried out in duplicate. For each replicate, cDNA from a single pool of oocytes or embryos was subdivided to amplify all genes during the same PCR assay. Templates used as negative controls in the PCR assay included an RT reaction lacking reverse transcriptase and $\mathrm{H}_{2} \mathrm{O}$. PCR was run on an MyiQ single color real-time thermal cycler (Bio-Rad). The following program was used: initial denaturation at $94{ }^{\circ} \mathrm{C}$ for $5 \mathrm{~min}$ followed by 40 cycles of $5 \mathrm{~s}$ at $94^{\circ} \mathrm{C}, 30 \mathrm{~s}$ at $60^{\circ} \mathrm{C}$, and $30 \mathrm{~s}$ at $72{ }^{\circ} \mathrm{C}$. Real-time fluorescence data were collected during the extension time. A melting curve was produced to verify individual PCR amplicons. Products were further confirmed on an agarose gel. Products from the first replicate were sequenced to verify identity.

\section{Quantification of transcripts levels}

For the expression pattern of SNF2-type ATPases, transcript levels of all genes were quantified using the relative quantification method based on comparative threshold cycles values $\left(C_{\mathrm{T}}\right)$. The abundance of each single gene was determined relative to the abundance of the housekeeping gene YWHAG. YWHAG was chosen as normalizer gene since it had been previously shown to maintain a constant level of expression throughout cleavage development in porcine embryos (Whitworth et al. 2005). The $C_{\mathrm{T}}$ value, or the cycle number during the log-linear phase of the PCR at which time the amount of detected PCR product rises above background levels, was determined for each reaction. Briefly, the $C_{\mathrm{T}}$ value for each single gene was subtracted from the $C_{\mathrm{T}}$ value for $Y W H A G$ to obtain a change in $C_{\mathrm{T}}\left(\Delta C_{\mathrm{T}}\right)$. To analyze the relative amount of SNF2-type ATPases in the same-stage embryos, we compare $\Delta C_{\mathrm{T}}$. To analyze the relative amount of SMARCA2, SMARCA4, SMARCA5, CDH3, and CHD5 during embryo development, we compared fold differences relative to the GV stage (GV stage as calibrator); for analysis of the relative amount of SMARCA1, comparisons were made relative to the MII-stage oocytes (MII stage as calibrator). Fold differences were calculated assuming an amplification efficiency of $100 \%$ and using the equation $2^{-\left(\Delta \Delta C_{T}\right)}$. To analyze the relative abundance of all genes following injection, we compared fold difference using the non-injected control as calibrator.

\section{Statistical analysis}

For quantitative PCR, $2^{-\left(\Delta \Delta C_{\mathrm{T}}\right)}$ values were imported into SAS (SAS Institute Inc., Cary, NC, USA) and analyzed with a GLM one-way ANOVA procedure. The model incorporated stage and replicate as the main factors. For the expression pattern of SNF2-type ATPases, a multiple comparisons ranking using Tukey's post-test was performed and $P<0.05$ was considered significant. For the microinjection experiment, a multiple comparison ranking using the LSD post-test was performed and $P<0.05$ was considered significant. Analysis of the proportion of embryos developing to the two-cell, four-cell, and blastocyst stages were performed using a logistic regression model and was analyzed with PROC GENMODE in SAS. For nuclei count analysis, data were transformed with a log transformation and analyzed with a two-way ANOVA with replicate and treatments as the main factors.

\section{Declaration of interest}

The authors declare no conflicts of interest that would prejudice the impartiality of the research reported here.

\section{Funding}

This work was supported by the office of Agricultural Research Programs, Purdue University (ARP manuscript no. 2008-18348).

\section{Acknowledgements}

We wish to thank the Indiana Packers Corporation for the providing the porcine ovaries used in this study. We thank Dr Anthony Imbalzano for the kind gift of SMARCA2 and SMARCA4 constructs. We also thank Dr Randall S Prather, University of Missouri-Columbia, for providing us with the YWHAG construct for use in our PCR experiments. 


\section{References}

Abeydeera LR \& Day BN 1997 Fertilization and subsequent development in vitro of pig oocytes inseminated in a modified tris-buffered medium with frozen-thawed ejaculated spermatozoa. Biology of Reproduction $\mathbf{5 7}$ 729-734.

Abeydeera LR, Wang WH, Prather RS \& Day BN 1998 Maturation in vitro of pig oocytes in protein-free culture media: fertilization and subsequent embryo development in vitro. Biology of Reproduction $\mathbf{5 8}$ 1316-1320.

Armstrong L, Lako M, Dean W \& Stojkovic M 2006 Epigenetic modification is central to genome reprogramming in somatic cell nuclear transfer. Stem Cells 24 805-814.

Bandyopadhyay D, Curry JL, Lin Q, Richards HW, Chen D, Hornsby PJ, Timchenko NA \& Medrano EE 2007 Dynamic assembly of chromatin complexes during cellular senescence: implications for the growth arrest of human melanocytic nevi. Aging Cell 6 577-591.

Banine F, Bartlett C, Gunawardena R, Muchardt C, Yaniv M, Knudsen ES, Weissman BE \& Sherman LS 2005 SWI/SNF chromatin-remodeling factors induce changes in DNA methylation to promote transcriptional activation. Cancer Research 65 3542-3547.

Batsché E, Yaniv M \& Muchardt C 2006 The human SWI/SNF subunit Brm is a regulator of alternative splicing. Nature Structural and Molecular Biology 13 22-29.

Bortvin A, Eggan K, Skaletsky H, Akutsu H, Berry DL, Yanagimachi R, Page DC \& Jaenisch R 2003 Incomplete reactivation of Oct4-related genes in mouse embryos cloned from somatic nuclei. Development 130 1673-1680.

Bowen NJ, Fujita N, Kajita M \& Wade PA 2004 Mi-2/NuRD: multiple complexes for many purposes. Biochimica et Biophysica Acta 1677 52-57.

Bultman S, Gebuhr T, Yee D, La Mantia C, Nicholson J, Gilliam A, Randazzo F, Metzger D, Chambon P, Crabtree G et al. 2000 A BRG1 null mutation in the mouse reveals functional differences among mammalian SWI/SNF complexes. Molecular Cell 6 1287-1295.

Bultman SJ, Gebuhr TC, Pan H, Svoboda P, Schultz RM \& Magnuson T 2006 Maternal $B R G 1$ regulates zygotic genome activation in the mouse. Genes and Development 20 1744-1754.

Choudhary P \& Varga-Weisz P 2007 ATP-dependent chromatin remodelling: action and reaction. Sub-Cellular Biochemistry 41 29-43.

Dean W, Santos F, Stojkovic M, Zakhartchenko V, Walter J, Wolf E \& Reik W 2001 Conservation of methylation reprogramming in mammalian development: aberrant reprogramming in cloned embryos. PNAS 98 13734-13738.

Dunaief JL, Strober BE, Guha S, Khavari PA, Alin K, Luban J, Begemann M, Crabtree GR \& Goff SP 1994 The retinoblastoma protein and BRG1 form a complex and cooperate to induce cell cycle arrest. Cell 7 119-130.

Fulka H, Mrazek M, Tepla O \& Fulka J Jr 2004 DNA methylation pattern in human zygotes and developing embryos. Reproduction 128 703-708.

Gangaraju VK \& Bartholomew B 2007 Mechanisms of ATP dependent chromatin remodeling. Mutation Research 618 3-17.

Hall JA \& Georgel PT 2007 CHD proteins: a diverse family with strong ties. Biochemistry and Cell Biology 85 463-476.

Hattori N, Imao Y, Nishino K, Hattori N, Ohgane J, Yagi S, Tanaka S \& Shiota K 2007 Epigenetic regulation of Nanog gene in embryonic stem and trophoblast stem cells. Genes to Cells 12 387-396.

van der Heijden GW, Dieker JW, Derijck AA, Muller S, Berden JH, Braat DD, van der Vlag J \& de Boer P 2005 Asymmetry in histone H3 variants and lysine methylation between paternal and maternal chromatin of the early mouse zygote. Mechanisms of Development 122 1008-1022.

Hildmann C, Riester D \& Schwienhorst A 2007 Histone deacetylases - an important class of cellular regulators with a variety of functions. Applied Microbiology and Biotechnology 75 487-497.

Inoue K, Ogonuki N, Miki H, Hirose M, Noda S, Kim JM, Aoki F, Miyoshi H \& Ogura A 2006 Inefficient reprogramming of the hematopoietic stem cell genome following nuclear transfer. Journal of Cell Science 119 1985-1991.

Ito $\mathrm{T}$, Watanabe $\mathrm{H}$, Yamamichi $\mathrm{N}$, Kondo $\mathrm{S}$, Tando $\mathrm{T}$, Haraguchi $\mathrm{T}$, Mizutani T, Sakurai K, Fujita S, Izumi T, Isobe T et al. 2008 Brm transactivates the telomerase reverse transcriptase (TERT) gene and modulates the splicing patterns of its transcripts in concert with p54(nrb). Biochemical Journal 411 201-209.
Itoh T, Miyake K \& lijima S 2008 Differentiation-specific expression of chromatin remodeling factor BRM. Biochemical and Biophysical Research Communications 366 827-833.

Jarrell VL, Day BN \& Prather RS 1991 The transition from maternal to zygotic control of development occurs during the 4-cell stage in the domestic pig, Sus scrofa: quantitative and qualitative aspects of protein synthesis. Biology of Reproduction 44 62-68.

Kondo T \& Raff M 2004 Chromatin remodeling and histone modification in the conversion of oligodendrocyte precursors to neural stem cells. Genes and Development 18 2963-2972.

Konev AY, Tribus M, Park SY, Podhrasky V, Lim CY, Emelyanov AV, Vershilova E, Pirrotta V, Kadonaga JT, Lusser A et al. 2007 CHD1 motor protein is required for deposition of histone variant $\mathrm{H} 3.3$ into chromatin in vivo. Science 317 1087-1090.

Kumar BM, Jin HF, Kim JG, Ock SA, Hong Y, Balasubramanian S, Choe SY \& Rho GJ 2007 Differential gene expression patterns in porcine nuclear transfer embryos reconstructed with fetal fibroblasts and mesenchymal stem cells. Developmental Dynamics 236 435-446.

Kuzmichev A, Zhang Y, Erdjument-Bromage H, Tempst P \& Reinberg D 2002 Role of the Sin3-histone deacetylase complex in growth regulation by the candidate tumor suppressor p33(ING1). Molecular and Cellular Biology 22 835-848.

Kwon CS \& Wagner D 2007 Unwinding chromatin for development and growth: a few genes at a time. Trends in Genetics 23 403-412.

Latham KE, Solter D \& Schultz RM 1992 Acquisition of a transcriptionally permissive state during the 1-cell stage of mouse embryogenesis. Developmental Biology 149 457-462.

Lazzaro MA \& Picketts DJ 2001 Cloning and characterization of the murine Imitation Switch (ISWI) genes: differential expression patterns suggest distinct developmental roles for Snf2h and SNF2L. Journal of Neurochemistry 77 1145-1156.

Lazzaro MA, Pépin D, Pescador N, Murphy BD, Vanderhyden BC \& Picketts DJ 2006 The imitation switch protein SNF2L regulates steroidogenic acute regulatory protein expression during terminal differentiation of ovarian granulosa cells. Molecular Endocrinology 20 2406-2417.

LeGouy E, Thompson EM, Muchardt C \& Renard JP 1998 Differential preimplantation regulation of two mouse homologues of the yeast SWI2 protein. Developmental Dynamics 212 38-48.

Lin T, Chao C, Saito S, Mazur SJ, Murphy ME, Appella E \& Xu Y 2005 p53 induces differentiation of mouse embryonic stem cells by suppressing Nanog expression. Nature Cell Biology 7 165-171.

Liu H, Kim JM \& Aoki F 2004 Regulation of histone H3 lysine 9 methylation in oocytes and early pre-implantation embryos. Development 131 2269-2280.

Magnani L \& Cabot RA 2007 Developmental arrest induced in cleavage stage porcine embryos following microinjection of mRNA encoding BRAHMA (Smarca 2), a chromatin remodeling protein. Molecular Reproduction and Development 74 1262-1267.

Magnani L \& Cabot RA 2008 In vitro and in vivo derived porcine embryos possess similar, but not identical, patterns of Oct4, Nanog, and Sox 2 mRNA expression during cleavage development. Molecular Reproduction and Development. 75 1726-1735

Magnani L, Lee K, Fodor WL, Machaty Z \& Cabot RA 2008 Developmental capacity of porcine nuclear transfer embryos correlate with levels of chromatin-remodeling transcripts in donor cells. Molecular Reproduction and Development 75 766-776.

Marfella CG, Ohkawa Y, Coles AH, Garlick DS, Jones SN \& Imbalzano AN 2006 Mutation of the SNF2 family member Chd2 affects mouse development and survival. Journal of Cellular Physiology 209 162-171.

McGraw S, Robert C, Massicotte L \& Sirard M 2003 Quantification of histone acetyltransferase and histone deacetylase transcripts during early bovine embryo development. Biology of Reproduction 68 383-389.

Monk M, Boubelik M \& Lehnert S 1987 Temporal and regional changes in DNA methylation in the embryonic, extraembryonic and germ cell lineages during mouse embryo development. Development 99 371-382.

Muchardt C \& Yaniv M 1993 A human homologue of Saccharomyces cerevisiae SNF2/SWI2 and Drosophila brm genes potentiates transcriptional activation by the glucocorticoid receptor. EMBO Journal 12 4279-4290.

Muchardt C \& Yaniv M 2001 When the SWI/SNF complex remodels the cell cycle. Oncogene 20 3067-3075.

Mujtaba S, Zeng L \& Zhou MM 2007 Structure and acetyl-lysine recognition of the bromodomain. Oncogene 26 5521-5527. 
Ng RK \& Gurdon JB 2008 Epigenetic memory of an active gene state depends on histone $\mathrm{H} 3.3$ incorporation into chromatin in the absence of transcription. Nature Cell Biology 10 102-109.

Pal S, Yun R, Datta A, Lacomis L, Erdjument-Bromage H, Kumar J, Tempst P \& Sif S $2003 \mathrm{mSin} 3 \mathrm{~A} /$ histone deacetylase 2- and PRMT5-containing $B R G 1$ complex is involved in transcriptional repression of the Myc target gene cad. Molecular and Cellular Biology 23 7475-7487.

Reik W, Dean W \& Walter J 2001 Epigenetic reprogramming in mammalian development. Science 293 1089-1093.

Reisman DN, Strobeck MW, Betz BL, Sciariotta J, Funkhouser W Jr, Murchardt C, Yaniv M, Sherman LS, Knudsen ES \& Weissman BE 2002 Concomitant down-regulation of BRM and BRG1 in human tumor cell lines: differential effects on RB-mediated growth arrest vs CD44 expression. Oncogene 21 1196-1207.

Ruau D, Ensenat Waser R, Dinger TC, Vallabhapurapu DS, Rolletschek A, Hacker C, Hieronymus T, Wobus AM, Müller AM \& Zenke M 2008 Pluripotency associated genes are reactivated by chromatin modifying agents in neurosphere cells. Stem Cells 26 920-926.

Seo S, Richardson GA \& Kroll KL 2005 The SWI/SNF, chromatin remodeling protein $B R G 1$ is required for vertebrate neurogenesis and mediates transactivation of Ngn and NeuroD. Development 132 105-115.

de la Serna IL, Ohkawa Y \& Imbalzano AN 2006 Chromatin remodelling in mammalian differentiation: lessons from ATP-dependent remodellers. Nature Reviews Genetics 7 461-473.

de la Serna IL, Carlson KA, Hill DA, Guidi CJ, Stephenson RO, Sif S, Kingston RE \& Imbalzano AN 2000 Mammalian SWI-SNF complexes contribute to activation of the hsp70 gene. Molecular and Cellular Biology 20 2839-2851.

Shanahan F, Seghezzi W, Parry D, Mahony D \& Lees E 1999 Cyclin E associates with BAF155 and BRG1, components of the mammalian SWISNF complex, and alters the ability of $B R G 1$ to induce growth arrest. Molecular and Cellular Biology 19 1460-1469.

Sif S 2004 ATP-dependent nucleosome remodeling complexes: enzymes tailored to deal with chromatin. Journal of Cellular Biochemistry 91 1087-1098.

Sif S, Saurin AJ, Imbalzano AN \& Kingston RE 2001 Purification and characterization of $\mathrm{mSin} 3 \mathrm{~A}$-containing BRG1 and hBrm chromatin remodeling complexes. Genes and Development 15 603-618.

Sikorska M, Sandhu JK, Deb-Rinker P, Jezierski A, Leblanc J, Charlebois C, Ribecco-Lutkiewicz M, Bani-Yaghoub M \& Walker PR 2008 Epigenetic modifications of SOX2 enhancers, SRR1 and SRR2, correlate with in vitro neural differentiation. Journal of Neuroscience Research 86 1680-1693.

De Sousa PA, Caveney A, Westhusin ME \& Watson AJ 1998 Temporal patterns of embryonic gene expression and their dependence on oogenetic factors. Theriogenology 49 115-128.

Stopka T \& Skoultchi Al 2003 The ISWI ATPase Snf2h is required for early mouse development. PNAS 100 14097-14102.

Strobeck MW, Knudsen KE, Fribourg AF, DeCristofaro MF, Weissman BE, Imbalzano AN \& Knudsen ES 2000 BRG-1 is required for RB-mediated cell cycle arrest. PNAS 97 7748-7753.

Strober BE, Dunaief JL, Guha P \& Goff SP 1996 Functional interactions between the hBRM/hBRG1 transcriptional activators and the pRB family of proteins. Molecular and Cellular Biology 16 1576-1583.
Torres-Padilla ME \& Zernicka-Goetz M 2006 Role of TIF1alpha as a modulator of embryonic transcription in the mouse zygote. Journal of Cell Biology 174 329-338.

Torres-Padilla ME, Bannister AJ, Hurd PJ, Kouzarides T \& ZernickaGoetz M 2006 Dynamic distribution of the replacement histone variant H3.3 in the mouse oocyte and preimplantation embryos. International Journal of Developmental Biology 50 455-461.

Torres-Padilla ME, Parfitt DE, Kouzarides T \& Zernicka-Goetz M 2007 Histone arginine methylation regulates pluripotency in the early mouse embryo. Nature 445 214-218.

Trouche D, Le Chalony C, Muchardt C, Yaniv M \& Kouzarides T 1997 RB and hbrm cooperate to repress the activation functions of E2F1. PNAS 94 11268-11273.

Underhill C, Qutob MS, Yee SP \& Torchia J 2000 A novel nuclear receptor corepressor complex, N-CoR, contains components of the mammalian SWI/SNF complex and the corepressor KAP-1. Journal of Biological Chemistry 275 40463-40470.

Whitworth KM, Agca C, Kim JG, Patel RV, Springer GK, Bivens NJ, Forrester LJ, Mathialagan N, Green JA \& Prather RS 2005 Transcriptional profiling of pig embryogenesis by using a $15-\mathrm{K}$ member unigene set specific for pig reproductive tissues and embryos. Biology of Reproduction 72 1437-1451.

Wiekowski M, Miranda M, Nothias JY \& DePamphilis ML 1997 Changes in histone synthesis and modification at the beginning of mouse development correlate with the establishment of chromatin mediated repression of transcription. Journal of Cell Science 110 1147-1158.

Wuensch A, Habermann FA, Kurosaka S, Klose R, Zakhartchenko V, Reichenbach HD, Sinowatz F, McLaughlin KJ \& Wolf E 2007 Quantitative monitoring of pluripotency gene activation after somatic cloning in cattle. Biology of Reproduction 76 983-991.

Xie Y, Sun T, Wang QT, Wang Y, Wang F, Puscheck E \& Rappolee DA 2005 Acquisition of essential somatic cell cycle regulatory protein expression and implied activity occurs at the second to third cell division in mouse preimplantation embryos. FEBS Letters 579 398-408.

Yoshioka K, Suzuki C, Tanaka A, Anas IM \& Iwamura S 2002 Birth of piglets derived from porcine zygotes cultured in a chemically defined medium. Biology of Reproduction 66 112-119.

Zhang HS, Gavin M, Dahiya A, Postigo AA, Ma D, Luo RX, Harbour JW \& Dean DC 2000 Exit from G1 and S phase of the cell cycle is regulated by repressor complexes containing HDAC-Rb-hSWI/SNF and Rb-hSWI/SNF. Cell 101 79-89.

Zheng P, Patel B, McMenamin M, Paprocki AM, Schramm RD, Nagl NG Jr, Wilsker D, Wang X, Moran E \& Latham KE 2004 Expression of genes encoding chromatin regulatory factors in developing rhesus monkey oocytes and preimplantation stage embryos: possible roles in genome activation. Biology of Reproduction 70 1419-1427.

Received 6 August 2008

First decision 15 September 2008

Accepted 8 October 2008 